\title{
Is Nasal Packing Essential after Functional Endoscopic Sinus Surgery? A Randomized, Controlled Trial
}

\author{
${ }^{1}$ Raghav Mehan, ${ }^{2}$ Lalee Varghese, ${ }^{3}$ Regi Kurien, ${ }^{4}$ Visalakshi Jeyaseelan, ${ }^{5}$ Vedantam Rupa
}

\begin{abstract}
Aim: To assess the need for nasal packing following functional endoscopic sinus surgery (FESS) for chronic rhinosinusitis (CRS).

Materials and methods: We used a prospective, singleblinded, intrapatient, randomized, controlled study design. Fifty patients undergoing bilateral FESS were randomized to have nasal packing on one side for a day and no packing on the other. Postoperative bleeding, nasal block, facial pain, and headache were monitored for 5 days. Improvement of symptoms and endoscopic and sino-nasal outcome test (SNOT)-22 scores at the 4 th and 12 th postoperative weeks were noted.

Results: Postoperative bleeding was significantly more in the no-packing side on the first postoperative day $(p<0.002)$ and, on the packed side, after pack removal on the second $(p<0.001)$ and third days $(p=0.003)$, with no difference thereafter. Three patients developed postoperative bleeding on the unpacked side necessitating packing in the recovery room. Nasal block $(p<0.001)$ and pain $(p<0.001)$ were worse on the packed side for the first 2 days. Both intention-to-treat and per-protocol analysis showed no significant difference between the two groups for all parameters.
\end{abstract}

Conclusion: Nasal packing following routine FESS is not required for most patients to prevent either postoperative bleeding or poor surgical outcome.

Clinical significance: Most ear-nose-throat (ENT) surgeons in India tend to pack the nose for at least 1 day postoperatively. The procedure of nasal packing and its removal may itself cause mucosal trauma, which may lead to delayed healing and increased risk of scarring and synechiae formation. By using hypotensive anesthesia, there is less intraoperative bleeding, and postoperative nasal packing can be avoided totally or minimized. Our study has shown that the results of packing and leaving the nose unpacked are comparable both in terms of early postoperative morbidity as well as late sequelae and quality-of-life.

Keywords: Endoscopic, Nasal pack, Randomized trial, Sinus surgery.

\footnotetext{
${ }^{1}$ Postdoctoral Rhinology Fellow, ${ }^{2,3}$ Associate Professor ${ }^{4}$ Lecturer, ${ }^{5}$ Professor

1-3,5 Department of ENT, Christian Medical College, Vellore, Tamil Nadu, India

${ }^{4}$ Department of Biostatistics, Christian Medical College, Vellore Tamil Nadu, India
}

Corresponding Author: Lalee Varghese, Associate Professor Department of ENT, Christian Medical College, Vellore, Tamil Nadu, India, Phone: +919488636020, e-mail: laleevarghese@ yahoo.co.in
How to cite this article: Mehan R, Varghese L, Kurien $R$, Jeyaseelan V, Rupa V. Is Nasal Packing Essential after Functional Endoscopic Sinus Surgery? A Randomized, Controlled Trial. Clin Rhinol An Int J 2017;10(3):113-119.

Source of support: Nil

Conflict of interest: None

\section{INTRODUCTION}

Nasal packing after FESS is performed both to control postoperative hemorrhage and to prevent aspiration of blood in the postoperative period. In India, it is a frequent practice to tightly pack the nasal cavities after routine sinus surgery for at least a day and often for up to 3 to 5 days. Many surgeons routinely pack the nose irrespective of whether there has been excessive bleeding intraoperatively or not. Problems associated with postoperative nasal packing include nasal obstruction, postnasal discharge, headache, pain in the nose, epiphora and dry mouth, all of which significantly affect the quality-of-life. ${ }^{1,2}$ Although rare, the risk of toxic shock syndrome in patients who have a nasal pack in situ for a prolonged period exists. ${ }^{2,3}$ Removal of the nasal pack causes nasal pain and bleeding and makes the patient anxious. ${ }^{4,5}$ Studies have shown that patients consider the removal of a nasal pack as the most unpleasant part of the perioperative experience. ${ }^{6,7}$ The procedure of nasal packing and its removal may itself cause mucosal trauma, which may lead to delayed healing and increased risk of scarring and synechiae formation. ${ }^{8}$

Various materials are currently used for nasal packing. ${ }^{9-11}$ These include nonabsorbable nasal packs like cotton ribbon gauze, polyvinyl alcohol (PVA) sponge, silastic balloon packs, and Telfa as well as absorbable packs like gelatin sponge, synthetic polyurethane foam, carboxymethyl cellulose, and hyaluronic acid. Many centers in India still use the traditional method of anterior nasal packing using cotton ribbon gauze soaked with liquid paraffin or impregnated with antibiotic ointment like Neosporin or bismuth iodoform paraffin paste (BIPP). This type of nasal packing causes significant patient discomfort and mucosal trauma and is preferably avoided, especially after FESS, since they can lead to pressure necrosis, epistaxis upon removal, paraffin granulomas, and discomfort during placement and removal. Removal of a BIPP pack requires hours of soakage with liquid 
paraffin to facilitate the ease of removal. Currently, PVA packs are the most widely used the world over, $8,9,11$ but, in India, because of costs and lack of availability of this type of pack in many centers, BIPP packs or Vaseline gauze packs continue to be used.

While packing of the nose following FESS is routine for many surgeons, there are a few ${ }^{6,8,12}$ who have questioned this practice. There are very few properly designed trials on the subject, however. We are not aware of any such study performed in India. In order to assess the need for postoperative nasal packing after FESS, we, therefore, conducted a randomized, controlled trial to shed light on this controversial subject.

\section{MATERIALS AND METHODS}

\section{Study Design and Objectives}

A prospective, single-blinded, intrapatient, randomized controlled trial study design was employed comparing PVA nasal packing with no packing in the same patient after bilateral FESS for CRS. The primary objective was to compare postoperative bleeding between the packed and unpacked sides. There were two secondary objectives. The first was to compare postoperative nasal blockage, nasal pain, and lateralized headache between the packed and unpacked sides. The second was to compare the change in symptom and quality-of-life (SNOT-22) scores as well as postoperative endoscopic findings between the packed and unpacked sides.

\section{Methodology}

Patients presenting to the ENT department at our tertiary care center from February 2015 to February 2016 were enrolled after obtaining written informed consent. Patients aged $\geq 18$ years with bilateral CRS with or without polyposis were included in the study. The Lund-MacKay computed tomography (CT) scan score ${ }^{13}$ was used to assess the degree of sinus involvement, and only patients with a CT score difference of $\leq 3$ between sides were included in the study.

\section{Exclusion Criteria}

Patients with a previous history of endoscopic sinus surgery, uncontrolled hypertension or diabetes mellitus, hemoglobin concentration of $<10 \mathrm{gm} / \mathrm{dL}$, bleeding diathesis, systemic disease affecting the nose, and pregnant women were excluded.

\section{Preoperative Assessment and Surgery}

Symptoms and endoscopic findings were recorded using the Lund and Kennedy ${ }^{13}$ symptom and endoscopic score.
The SNOT-22 questionnaire ${ }^{14}$ was administered for quality-of-life assessment.

All patients underwent bilateral FESS under hypotensive general anesthesia. Both nasal cavities were packed with cottonoids soaked in 1:10,000 adrenaline-saline solution for 5 minutes followed by infiltration with $2 \%$ lignocaine and 1:1,00,000 adrenaline. Surgery was performed using the Messerklinger technique. Intraoperative hemostasis was secured with topical vasoconstrictor and bipolar cautery. Intraoperative bleeding was graded according to Boezaart and colleagues. ${ }^{15}$ After completing the surgery and achieving complete hemostasis, the patient was observed for 5 minutes to look for any further bleeding.

The sealed envelope (which was prepared in advance by the biostatistician using a block randomization technique) indicating the side to be packed was then opened. Accordingly, one side was packed with PVA sponge tampons placed in the middle meatus and floor of nose, while the other side was temporarily packed with cottonoid soaked in 1:10,000 adrenaline-saline solution, which was removed in the recovery room, before shifting the patient to the ward. Temporary packing was done to prevent oozing of blood, which could potentially occur during extubation.

The actual blood loss during surgery was calculated separately for each nasal cavity by ensuring that a separate suction tip and suction apparatus and separate cottonoids were used for each nasal cavity. Total fluid volume in the suction bottle excluding the volume of saline and saline-adrenaline solution plus the volume of blood soaked in cottonoids (i.e., total weight of cottonoids soaked with blood minus the total dry weight of cottonoids) was calculated separately for each nasal cavity and noted.

\section{Postoperative Care}

Saline nasal douching was started on the unpacked side as soon as the patient was fully awake. If any patient had bleeding from the side that was left unpacked, which did not stop within 5 minutes of removal of the temporary pack in the recovery room, a PVA sponge pack was inserted in that nasal cavity. All data were collected even for this group of "crossover" patients.

The nasal pack was removed the next morning by a surgeon who was different from the one who had performed the surgery. After discharge, the patient was advised oral antibiotics for 5 days, nasal saline douches every 2 hours for 3 weeks and thrice daily saline sprays for 3 months. In those patients with sinonasal allergy, fluticasone nasal spray two puffs in each nasal cavity once daily was prescribed for a period of 3 months. 


\section{Assessment of Subjective and Endoscopic Outcomes}

The primary outcome was early postoperative bleeding, which was noted by the on-call doctor in the ward and by the patient in a proforma from the day of discharge until the first postoperative visit on the 5th to 7 th day. Nasal bleeding was scored on a scale of 0 to 2 where $0=$ no bleeding, 1 = spotting of gauze/traces of clotted blood in the vestibule, and 2 = continuous bleeding (anterior or postnasal bleed).

The secondary outcome assessed was improvement in Lund-Kennedy symptom and endoscopic scores at 1 , 4, and 12 weeks after surgery as well as SNOT-22 qualityof-life scores at 12 weeks after surgery. Symptoms of nasal blockage, pain, and lateralized headache were scored on each side in a proforma by the patient using a numerical scale. Nasal blockage, pain, and lateralized headache were scored on a scale of 0 to 3 where $0=$ no symptom, $1=$ mild, $2=$ moderate, and $3=$ severe symptom.

Endoscopic findings of synechiae (between middle turbinate and lateral wall), edema, mucopurulent discharge, granulations, crusting, and stenosis were noted and an endoscopic scoring system for postoperative assessment was used, which has been described before. ${ }^{12}$ This was performed by a surgeon other than the one who had operated on the patient.

\section{Statistical Analysis}

\section{Sample Size Calculation}

With $80 \%$ power and $5 \%$ level of significance, the required sample size to show that there was no difference between packing and no-packing groups after FESS was found to be 45 subjects. A total sample size of 50 subjects was taken, allowing for dropouts.

\section{Randomization and Data Entry}

Randomization of the side was performed by permuted block randomization technique with the random sequence generated using SAS 9.3 computer software. The ratio of left-to-right sinus randomization assignments was 1:1. Data were entered into a validated electronic database and analyzed by an independent biostatistician.

\section{Data Analysis}

Mean with standard deviation for baseline variables like endoscopic score, CT score, surgical score, surgical field bleeding score, and blood loss were calculated. The degree of nasal bleed, nasal block, nasal pain, and lateralized headache was compared using Wilcoxon rank sum test after calculating mean scores in both the groups. The mean scores for postoperative nasal endoscopic findings were calculated in both the groups and were compared using Wilcoxon rank sum test. The mean with standard deviation for surgical outcomes was calculated for both sides and compared using Wilcoxon rank sum test or independent t-test. The pre- and postsurgical outcomes were compared using signed rank test or paired t-test based on the distribution of the difference between the pre-operative and postoperative scores in the surgical outcomes.

\section{Institutional Review Board Approval and Clinical trials.gov Registration}

The study was approved by the Institutional Review Board and Ethics committee of the Christian Medical College, Vellore, India. The study was registered by the Clinical Trials Registry of India (CTRI No: CTRI/2015/01/005387) on Jan 13, 2015.

\section{RESULTS}

From February 2015 to May 2016, a total of 83 patients underwent bilateral FESS for CRS. Thirty-three patients were excluded because they did not meet the inclusion criteria. Fifty patients with a mean age of 36.8 years (18-72 years) were included in the study and randomized after obtaining informed consent (Flow Chart 1).

\section{Baseline Characteristics}

Of a total of 50 patients recruited, there were 37 male and 13 female patients. The most common symptoms were nasal discharge (94\%) and nasal obstruction (88\%). Headache (70\%), hyposmia (44\%), and facial pain (10\%) were other associated symptoms. There were four diabetic and five hypertensive patients. Both allocated groups were comparable on baseline variables (Table 1). The right nasal cavity was packed in exactly half the patients.

\section{Follow-up}

All patients presented for their first follow-up, 12 (24\%) came for their second follow-up, and 36 (72\%) came for their third follow-up.

\section{OUTCOME ANALYSIS}

\section{Primary Outcome Analysis: Early Postoperative Bleeding}

As three patients in the unpacked group developed epistaxis in the immediate postoperative period for which packing was required, both per-protocol analysis of outcomes of 47 patients who were never packed and intention-to-treat analysis of outcomes of all 50 patients 


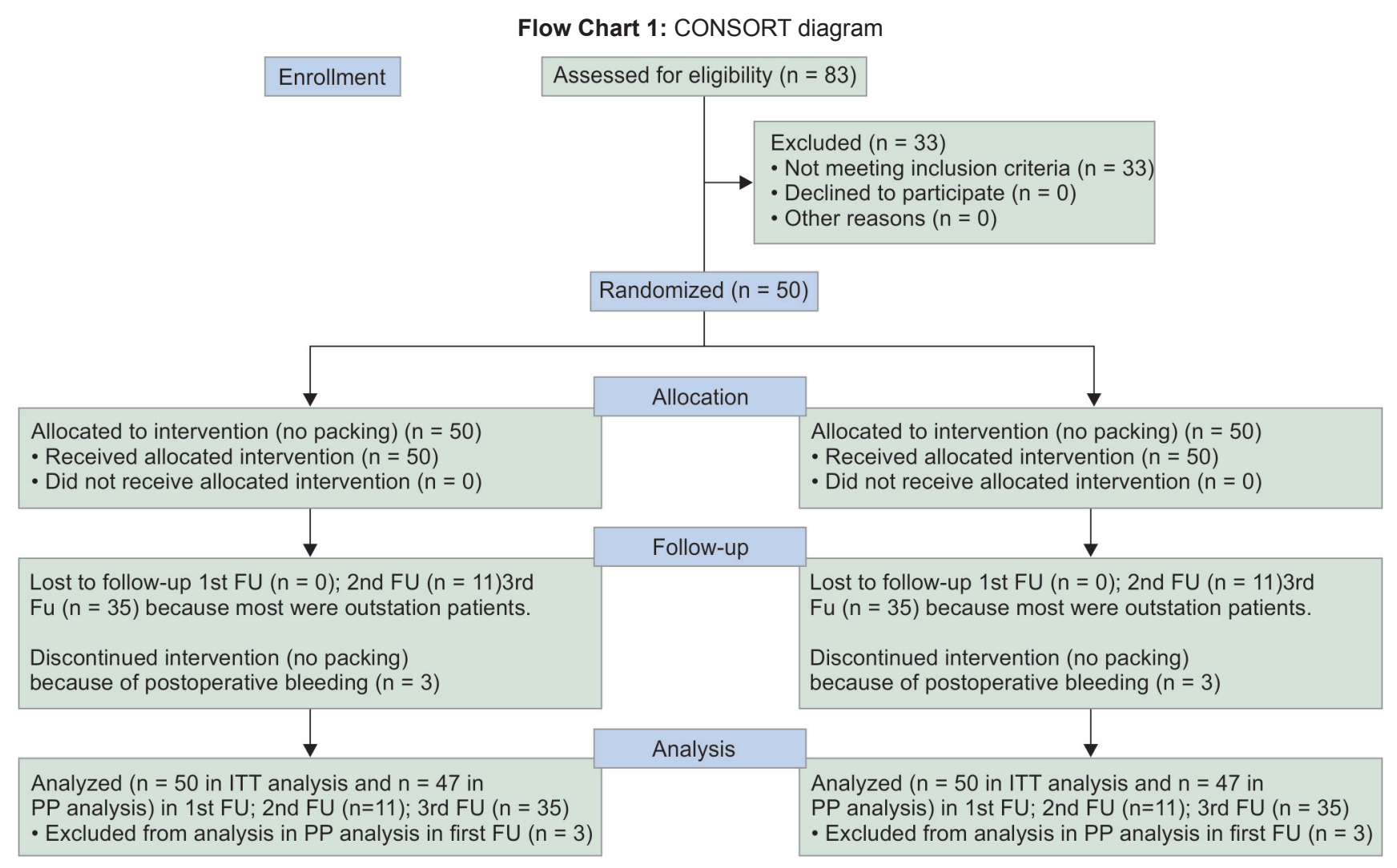

Table 1: Comparison of preoperative and intraoperative variables between no-packing and packing sides $(n=50)$

\begin{tabular}{llll}
\hline Variable & No packing & Packing & $p$-value \\
\hline Lund-Kennedy endoscopic score (mean \pm SD) & $3.72 \pm 1.39$ & $3.68 \pm 1.33$ & 0.88 \\
Lund-MacKay CT score (mean \pm SD) & $6.28 \pm 2.87$ & $6.38 \pm 2.63$ & 0.86 \\
Lund-MacKay surgery score (mean \pm SD) & $4.38 \pm 1.63$ & $4.22 \pm 1.62$ & 0.62 \\
Boezaart grading (mean \pm SD) & $2.46 \pm 0.65$ & $2.42 \pm 0.64$ & 0.76 \\
Intra-op blood loss (mean \pm SD, in $\mathrm{mL}$ ) & $60.6 \pm 47.54$ & $52.94 \pm 40.66$ & 0.39 \\
\hline
\end{tabular}

in the study group were performed. These two types of analysis were performed to avoid bias in interpretation of results because of nonrandom attrition of study participants due to crossover.

The per-protocol analysis with 47 patients (Table 2) showed that the degree of postoperative nasal bleeding was more $(p=0.002)$ in the no-packing side than the packing side on the first postoperative day. Bleeding was more on the packed side on the second $(p<0.001)$ and third ( $p=0.003$ ) postoperative days, however, following pack removal. There was no statistically significant difference in bleeding on postoperative days 4 and 5 .

The intention-to-treat analysis using all 50 patients (Table 3) showed similar results.

\section{Secondary Outcome Analysis}

\section{Early Postoperative Lund-Kennedy Symptom Score}

Both per-protocol analysis of 47 patients who were not packed and intention-to-treat analysis of all 50 patients were performed. Using per-protocol analysis (Table 2) and comparing the two arms, nasal block was significantly more in the packing side on the first and second postoperative days $(\mathrm{p}<0.001$ and $\mathrm{p}=0.004)$. Similar results were obtained with intention-to-treat analysis (Table 3).

Using per-protocol analysis (Table 2) and comparing the two arms, nasal pain was also significantly more in the packing side on the first and second postoperative days $(\mathrm{p}<0.001$ and $\mathrm{p}=0.004)$. Similar differences were obtained with intention-to-treat analysis (Table 3).

Headache was significantly more on the packing side on the first postoperative day alone $(p=0.006)$ using perprotocol analysis (Table 2) but not with intention-to-treat analysis.

\section{Late Postoperative Lund-Kennedy Symptom Score and SNOT-22 Score}

There was significant improvement in the LundKennedy postoperative symptom score at 4 and 12 weeks $(p<0.0001)$. There was also significant improvement in 
Is Nasal Packing Essential after Functional Endoscopic Sinus Surgery?

Table 2: Comparison of immediate postoperative symptom scores between no-packing and packing sides using per-protocol analysis $(n=47)$

\begin{tabular}{|c|c|c|c|}
\hline Symptom & $\begin{array}{l}\text { No packing } \\
\text { [Mean score } \pm \\
\text { SD (min, max)] }\end{array}$ & $\begin{array}{l}\text { Packing [Mean } \\
\text { score } \pm S D \\
(\min , \max )]\end{array}$ & $p$-value \\
\hline \multicolumn{4}{|c|}{$\begin{array}{l}\text { Nasal bleeding } \\
(0-2)\end{array}$} \\
\hline POD 1 & $0.36 \pm 0.49(0,1)$ & $0.09 \pm 0.28(0,1)$ & 0.002 \\
\hline POD 2 & $0.32 \pm 0.47(0,1)$ & $0.94 \pm 0.44(0,2)$ & $<0.001$ \\
\hline POD 3 & $0.34 \pm 0.52(0,2)$ & $0.68 \pm 0.47(0,1)$ & 0.003 \\
\hline POD 4 & $0.36 \pm 0.53(0,2)$ & $0.43 \pm 0.50(0,1)$ & 0.439 \\
\hline POD 5 & $0.28 \pm 0.50(0,2)$ & $0.34 \pm 0.48(0,1)$ & 0.317 \\
\hline \multicolumn{4}{|c|}{ Nasal block (0-3) } \\
\hline POD 1 & $1.04 \pm 0.88(0,3)$ & $3.00 \pm 0(3,3)$ & $<0.001$ \\
\hline POD 2 & $1.06 \pm 1.03(0,3)$ & $1.57 \pm 1.04(0,3)$ & 0.004 \\
\hline POD 3 & $1.3 \pm 1.18(0,3)$ & $1.40 \pm 1.01(0,3)$ & 0.524 \\
\hline POD 4 & $1.23 \pm 1.07(0,3)$ & $1.30 \pm 0.95(0,3)$ & 0.604 \\
\hline POD 5 & $1.21 \pm 0.93(0,3)$ & $1.15 \pm 0.88(0,3)$ & 0.673 \\
\hline \multicolumn{4}{|c|}{ Nasal pain (0-3) } \\
\hline POD 1 & $0.26 \pm 0.57(0,3)$ & $0.85 \pm 0.93(0,3)$ & $<0.001$ \\
\hline POD 2 & $0.36 \pm 0.64(0,2)$ & $0.72 \pm 0.85(0,3)$ & 0.004 \\
\hline POD 3 & $0.43 \pm 0.74(0,3)$ & $0.38 \pm 0.53(0,2)$ & 0.711 \\
\hline POD 4 & $0.40 \pm 0.74(0,3)$ & $0.32 \pm 0.52(0,2)$ & 0.380 \\
\hline POD 5 & $0.40 \pm 0.71(0,3)$ & $0.32 \pm 0.52(0,2)$ & 0.234 \\
\hline \multicolumn{4}{|c|}{$\begin{array}{l}\text { Lateralized } \\
\text { headache (0-3) }\end{array}$} \\
\hline POD 1 & $0.26 \pm 0.64(0,3)$ & $0.51 \pm 0.88(0,3)$ & 0.006 \\
\hline POD 2 & $0.28 \pm 0.62(0,3)$ & $0.34 \pm 0.73(0,3)$ & 0.332 \\
\hline POD 3 & $0.43 \pm 0.80(0,3)$ & $0.43 \pm 0.83(0,3)$ & 1.000 \\
\hline POD 4 & $0.51 \pm 0.80(0,3)$ & $0.55 \pm 0.86(0,3)$ & 0.666 \\
\hline POD 5 & $0.49 \pm 0.75(0,3)$ & $0.43 \pm 0.77(0,3)$ & 0.470 \\
\hline
\end{tabular}

SD: Standard deviation; POD: Postoperative day

the postoperative SNOT-22 score at 12 weeks $(\mathrm{p}<0.0001)$. Endoscopic findings of synechiae (between middle turbinate and lateral wall), edema, mucopurulent discharge, granulations, crusting, and stenosis were similar in both groups (Table 4).

\section{DISCUSSION}

Postoperative care after FESS has not been standardized across the world. Even in India, there is a great variability in the manner of packing of the nose postoperatively across the country. Many surgeons are wary of keeping the nose unpacked because of the possibility of postoperative bleeding in the ward for which repacking is required. We were curious to assess whether in the Indian setting, where factors like hot weather and cultural practices are important, it would be possible to leave the nose unpacked. With the use of good hypotensive anesthesia for routine FESS, it has become possible to ensure complete disease removal with minimal bleeding in most patients. In the present study, using a randomized, controlled study design, we have been able to demonstrate conclusively that
Table 3: Comparison of immediate postoperative symptom scores between no-packing and packing sides using intention-to-treat analysis $(n=50)$

\begin{tabular}{|c|c|c|c|}
\hline Symptom & $\begin{array}{l}\text { No packing } \\
{[\text { Mean score } \pm S D} \\
(\min , \max )]\end{array}$ & $\begin{array}{l}\text { Packing [Mean } \\
\text { score } \pm S D \\
(\min , \max )]\end{array}$ & $p$-value \\
\hline \multicolumn{4}{|l|}{$\begin{array}{l}\text { Nasal } \\
\text { bleeding } \\
(0-2)\end{array}$} \\
\hline POD 1 & $0.34 \pm 0.479(0,1)$ & $0.08 \pm 0.274(0,1)$ & 0.001 \\
\hline POD 2 & $0.34 \pm 0.519(0,2)$ & $0.88 \pm 0.480(0,1)$ & 0 \\
\hline POD 3 & $0.32 \pm 0.513(0,2)$ & $0.64 \pm 0.485(0,1)$ & 0.001 \\
\hline POD 4 & $0.34 \pm 0.519(0,2)$ & $0.40 \pm 0.495(0,1)$ & 0.457 \\
\hline POD 5 & $0.26 \pm 0.487(0,2)$ & $0.32 \pm 0.471(0,1)$ & 0.416 \\
\hline \multicolumn{4}{|c|}{$\begin{array}{l}\text { Nasal block } \\
(0-3)\end{array}$} \\
\hline POD 1 & $1.020 \pm 08919(0,3)$ & $2.82 \pm 0.7197(0,3)$ & 0 \\
\hline POD 2 & $1.040 \pm 1.0294(0,3)$ & $1.48 \pm 1.0736(0,3)$ & 0.038 \\
\hline POD 3 & $1.220 \pm 1.1830(0,4)$ & $1.32 \pm 1.0388(0,3)$ & 0.496 \\
\hline POD 4 & $1.160 \pm 1.0759(0,3)$ & $1.22 \pm 0.9750(0,3)$ & 0.659 \\
\hline POD 5 & $1.140 \pm 0.9478(0,3)$ & $1.080 \pm 0.8999(0,3)$ & 0.776 \\
\hline \multicolumn{4}{|l|}{$\begin{array}{l}\text { Nasal pain } \\
(0-3)\end{array}$} \\
\hline POD 1 & $0.28 \pm 0.607(0,3)$ & $0.80 \pm 0.926(0,3)$ & 0.002 \\
\hline POD 2 & $0.34 \pm 0.626(0,2)$ & $0.68 \pm 0.844(0,3)$ & 0.023 \\
\hline POD 3 & $0.40 \pm 0.728(0,3)$ & $0.38 \pm 0.530(0,2)$ & 0.647 \\
\hline POD 4 & $0.38 \pm 0.725(0,3)$ & $0.32 \pm 0.513(0,2)$ & 0.941 \\
\hline POD 5 & $0.38 \pm 0.697(0,3)$ & $0.30 \pm 0.505(0,2)$ & 0.850 \\
\hline \multicolumn{4}{|l|}{$\begin{array}{l}\text { Lateralized } \\
\text { headache } \\
(0-3)\end{array}$} \\
\hline POD 1 & $0.24 \pm 0.625(0,3)$ & $0.48 \pm 0.863(0,3)$ & 0.127 \\
\hline POD 2 & $0.26 \pm 0.600(0,3)$ & $0.36 \pm 0.749(0,3)$ & 0.684 \\
\hline POD 3 & $0.40 \pm 0.782(0,3)$ & $0.42 \pm 0.810(0,3)$ & 0.863 \\
\hline POD 4 & $0.48 \pm 0.789(0,3)$ & $0.52 \pm 0.839(0,3)$ & 0.838 \\
\hline POD 5 & $0.46 \pm 0.734(0,3)$ & $0.4 \pm 0.756(0,3)$ & 0.536 \\
\hline
\end{tabular}

SD: Standard deviation; POD: Postoperative day

there is no benefit to be gained by packing the nose after FESS for CRS.

A review of the literature showed that many authors have not found the need for postoperative nasal packing after routine sinus surgery, ${ }^{6,9,12}$ Bugten et $\mathrm{al}^{9}$ found no difference in postoperative epistaxis with or without nasal packing. Eliashar et $\mathrm{al}^{6}$ and Orlandi and Lanza ${ }^{12}$ showed that 92 and $87 \%$ patients respectively, did not require packing or a hemostatic agent after FESS. In the present study, we found that up to $94 \%$ of patients require no nasal packing following FESS. In contrast, Saedi et al ${ }^{11}$ found that there was a significant incidence of postoperative bleeding in those with unpacked noses ( $p=0.005)$.

Patients who have their noses packed frequently complain of significant nasal block, pain, and headache on the first 2 days after surgery. In the present study, all patients with nasal packs experienced these symptoms. Similar findings were noted by others. ${ }^{8}$ In the study by Bugten et al, ${ }^{9}$ however, despite having bilateral nonabsorbable 


\begin{tabular}{|c|c|c|c|}
\hline Endoscopic finding & $\begin{array}{l}\text { No packing [Mean score } \pm \\
\text { SD (min, max)] }\end{array}$ & $\begin{array}{l}\text { Packing [Mean score } \pm \\
\text { SD (min, max)] }\end{array}$ & $p$-value \\
\hline \multicolumn{4}{|l|}{ Synechia (0-3) } \\
\hline Postoperative 1 week & $0 \pm 0(0,0)$ & $0 \pm 0(0,0)$ & 1.0 \\
\hline Postoperative 4 weeks & $0.17 \pm 0.39(0,1)$ & $0.17 \pm 0.39(0,1)$ & 1.0 \\
\hline Postoperative 12 weeks & $0.25 \pm 0.5(0,2)$ & $0.25 \pm 0.44(0,1)$ & 1.0 \\
\hline \multicolumn{4}{|l|}{ Edema (0-3) } \\
\hline Postoperative 1 week & $0.04 \pm 0.2(0,1)$ & $0.04 \pm 0.2(0,1)$ & 1.0 \\
\hline Postoperative 4 weeks & $0.67 \pm 0.89(0,2)$ & $0.75 \pm 0.75(0,2)$ & 0.66 \\
\hline Postoperative 12 weeks & $0.44 \pm 0.77(0,3)$ & $0.64 \pm 0.99(0,3)$ & 0.08 \\
\hline \multicolumn{4}{|l|}{ Pus discharge (0-2) } \\
\hline Postoperative 1 week & $0.06 \pm 0.32(0,2)$ & $0.06 \pm 0.32(0,2)$ & 1.0 \\
\hline Postoperative 4 weeks & $0.08 \pm 0.29(0,1)$ & $0.25 \pm 0.62(0,2)$ & 0.32 \\
\hline Postoperative 12 weeks & $0.08 \pm 0.28(0,1)$ & $0.11 \pm 0.40(0,2)$ & 0.66 \\
\hline \multicolumn{4}{|l|}{ Granulation (0-3) } \\
\hline Postoperative 1 week & $0 \pm 0(0,0)$ & $0 \pm 0(0,0)$ & 1.0 \\
\hline Postoperative 4 weeks & $0 \pm 0(0,0)$ & $0 \pm 0(0,0)$ & 1.0 \\
\hline Postoperative 12 weeks & $0 \pm 0(0,0)$ & $0.03 \pm 0.17(0,1)$ & 0.32 \\
\hline \multicolumn{4}{|l|}{ Stenosis (0-2) } \\
\hline Postoperative 1 week & $0 \pm 0(0,0)$ & $0 \pm 0(0,0)$ & 1.0 \\
\hline Postoperative 4 weeks & $0.08 \pm 0.29(0,1)$ & $0 \pm 0(0,0)$ & 0.32 \\
\hline Postoperative 12 weeks & $0.03 \pm 0.17(0,1)$ & $0.03 \pm 0.17(0,1)$ & 1.0 \\
\hline \multicolumn{4}{|l|}{ Crust (0-2) } \\
\hline Postoperative 1 week & $0.49 \pm 0.55(0,2)$ & $0.53 \pm 0.58(0,2)$ & 0.32 \\
\hline Postoperative 4 weeks & $0.08 \pm 0.29(0,1)$ & $0.08 \pm 0.29(0,1)$ & 1.0 \\
\hline Postoperative 12 weeks & $0 \pm 0(0,0)$ & $0.11 \pm 0.46(0,2)$ & 0.16 \\
\hline \multicolumn{4}{|l|}{ Total points } \\
\hline Postoperative 1 week & $0.60 \pm 0.71(0,3)$ & $0.64 \pm 0.79(0,4)$ & 0.32 \\
\hline Postoperative 4 weeks & $1.08 \pm 1.31(0,4)$ & $1.25 \pm 1.14(0,3)$ & 0.74 \\
\hline Postoperative 12 weeks & $0.81 \pm 1.14(0,4)$ & $1.17 \pm 1.42(0,6)$ & 0.11 \\
\hline
\end{tabular}

nasal packs in the middle meatus for 5 days postoperatively, there was no significant difference in nasal congestion, pain, or headache between those patients and the group that had no nasal pack. The unpacked group had no significant increase in bleeding, either.

Pain on pack removal is also a common occurrence. Saedi et $\mathrm{al}^{11}$ who compared gelatin sponge nasal packing with no packing reported relatively severe pain during pack removal in the packed group (mean visual analog scale pain score of $61 \pm 3$ ). There was no reported increase in pain in the packed group in the study by Bugten et $\mathrm{al}^{9}{ }^{9}$ however.

The outcome of FESS depends to a large extent on the quality of mucosal healing after surgery. Mucosal healing is variable and dependent on several host and surgical factors. The influence of nasal packing on the development of postoperative synechiae and stenosis could be considerable. Mucosal trauma appears to be one of the prime causes for synechiae formation, and nasal packing with medicated cotton gauze can cause significant trauma to the mucosa. In comparison, gelatin sponge tampons can be inserted relatively atraumatically and because they swell when injected with saline, exert sufficient pressure without producing surface ulceration. It is possible that as we used only gelatin sponge tampons for packing in this study, no statistically significant difference was observed in the presence of synechiae, edema, or stenosis between the no-packing and packing sides. Similar results were noted by others. ${ }^{8,10,11}$ Some authors have suggested that nasal packing may reduce the risk of postoperative adhesions, however. ${ }^{9}$

We observed significant improvement in the LundKennedy symptom and endoscopic scores as well as SNOT-22 scores at postoperative follow-up, similar to other studies. ${ }^{11,16,17}$ A low incidence of synechiae, granulations, and stenosis contributes to improved outcomes following FESS. The present study, similar to other studies, $8,10,11$ showed that leaving the nose unpacked resulted in no greater prevalence of synechiae, granulations, or stenosis than in those patients whose noses had been packed.

\section{CONCLUSION}

The results of our study show there is no need to routinely pack the nasal cavities after FESS for CRS, if hypotensive anesthesia has been used. Packing may be reserved for the few who develop unexpected epistaxis in the 
postoperative period. In those whose nose is left unpacked, the reduction in postoperative discomfort, pain, and headache is significant. There is no evidence for reduction in late complications or improved quality-of-life from packing the nose. We conclude, therefore, that the usual practice of packing the nose at the end of surgery can be discontinued.

\section{CLINICAL SIGNIFICANCE}

Most surgeons pack the nose routinely after endoscopic sinus surgery. The procedure of nasal packing and its removal may itself cause mucosal trauma, which may lead to delayed healing and increased risk of scarring and synechiae formation. In the era of hypotensive anesthesia, which facilitates complete disease removal, our experience has been that the role of postoperative nasal packing is limited. Our study has shown that the results of packing and leaving the nose unpacked are comparable both in terms of early postoperative morbidity and late sequelae and quality-of-life.

\section{REFERENCES}

1. Weber R, Hochapfel F, Draf W. Packing and stents in endonasal surgery. Rhinology 2000 Jul;38(2):49-62.

2. Fairbanks DN. Complications of nasal packing. Otolaryngol Head Neck Surg 1986 Mar;94(3):412-415.

3. Abram AC, Bellian KT, Giles WJ, Gross CW. Toxic shock syndrome after functional endonasal sinus surgery: an all or none phenomenon? Laryngoscope 1994 Aug;104(8 Pt 1): 927-931.

4. Chheda N, Katz AE, Gynizio L, Singer AJ. The pain of nasal tampon removal after nasal surgery: a randomized control trial. Otolaryngol Head Neck Surg 2009 Feb;140(2):215-217.

5. Kuo MJ, Zeitoun H, Macnamara M, Wagstaff K, Carlin WV, Turner N. The use of topical $5 \%$ lignocaine ointment for the relief of pain associated with post-operative nasal packing. Clin Otolaryngol 1995 Aug;20(4):357-359.
6. Eliashar R, Gross M, Wohlgelernter J, Sichel JY. Packing in endoscopic sinus surgery: is it really required? Otolaryngol Head Neck Surg 2006 Feb;134(2):276-279.

7. von Schoenberg M, Robinson P, Ryan R. Nasal packing after routine nasal surgery-is it justified? J Laryngol Otol 1993 Oct;107(10):902-905.

8. Mo JH, Han DH, Shin HW, Cha W, Chang MY, Jin HR. No packing versus packing after endoscopic sinus surgery: pursuit of patients' comfort after surgery. Am J Rhinol 2008 Sep-Oct;22(5):525-528.

9. Bugten V, Nordgård S, Skogvoll E, Steinsvåg S. Effects of nonabsorbable packing in middle meatus after sinus surgery. Laryngoscope 2006 Jan;116(1):83-88.

10. Wee JH, Lee CH, Rhee CS, Kim JW. Comparison between Gelfoam packing and no packing after endoscopic sinus surgery in the same patients. Eur Arch Otorhinolaryngol 2012 Mar;269(3):897-903.

11. Saedi B, Sadeghi M, Farschi S. Effect of polyvinyl acetal sponge nasal packing on post-operative care of nasal polyposis patients: a randomised, controlled, partly blinded study. J Laryngol Otol 2012 Apr;126(4):380-384.

12. Orlandi RR, Lanza DC. Is nasal packing necessary following endoscopic sinus surgery? Laryngoscope 2004 Sep;114(9):1541-1544.

13. Lund VJ, Kennedy DW. Staging for rhinosinusitis. Otolaryngol Head Neck Surg 1997 Sep;117(3 Pt 2):S35-S40.

14. Hopkins C, Gillett S, Slack R, Lund VJ, Browne JP. Psychometric validity of the 22-item Sinonasal Outcome Test. Clin Otolaryngol 2009 Oct;34(5):447-454.

15. Boezaart AP, van der Merwe J, Coetzee A. Comparison of sodium nitroprusside- and esmolol-induced controlled hypotension for functional endoscopic sinus surgery. Can J Anaesth 1995 May;42(5 Pt 1):373-376.

16. Poetker DM, Smith TL. Adult chronic rhinosinusitis: surgical outcomes and the role of endoscopic sinus surgery. Curr Opin Otolaryngol Head Neck Surg 2007 Feb;15(1):6-9.

17. Djukic V, Dudvarski Z, Arsovic N, Dimitrijevic M, Janosevic L. Clinical outcomes and quality of life in patients with nasal polyposis after functional endoscopic sinus surgery. Eur Arch Otorhinolaryngol 2015 Jan;272(1):83-89. 